\title{
Erratum to: Measuring sequences of keystrokes with jsPsych: Reliability of response times and interkeystroke intervals
}

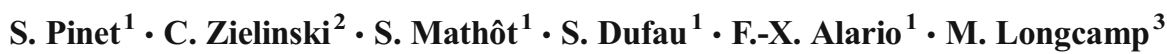

Published online: 24 March 2017

(C) Psychonomic Society, Inc. 2017

\section{Erratum to: Behav Res}

\section{DOI 10.3758/s13428-016-0776-3}

- The section entitled "Additional analyses" should be part of the "Results" section (same as subsection "Replication of original study" and both embedded in the Online experiment part of the paper) and not a separate section.

- Table 4: the "(Intercept)" row should not be part of the title row. Instead, it should be at the same level as "Hand" or "Sequence" (see Table 4).

- Similarly for Table 5: the "(Intercept)" row should not be part of the title row. Instead, it should be at the same level as "Hand" or "Sequence" (see Table 5).

- On Fig. 3 "RT \& IKI distributions" should not appear above the figures.
The online version of the original article can be found at doi:10.3758/ s13428-016-0776-3.
Table 4 Mixed model regression coefficient for RTs

\begin{tabular}{|c|c|c|c|c|c|}
\hline & $\beta$ & SE & $t$ & $95 \% \mathrm{CI}$ & (sig.) \\
\hline (Intercept) & 592.55 & 57.09 & 10.38 & & \\
\hline \multicolumn{6}{|c|}{ Experimental variables } \\
\hline Hand & -5.87 & 13.66 & -0.43 & {$[-32.69 ; 20.96]$} & \\
\hline Sequence & 46.71 & 5.43 & 8.61 & {$[36.05 ; 57.37]$} & $*$ \\
\hline Uncertainty & -116.48 & 3.34 & -34.90 & {$[-123.04 ;-109.92]$} & $*$ \\
\hline $\begin{array}{l}\text { Sequence } \mathrm{x} \\
\text { Uncertainty }\end{array}$ & -11.75 & 6.36 & -1.85 & {$[-24.24 ; 0.74]$} & \\
\hline Trial number & -1.05 & 0.03 & -31.65 & {$[-1.11 ;-0.98]$} & $*$ \\
\hline \multicolumn{6}{|l|}{ Subjects' variables } \\
\hline Gender & -54.32 & 13.78 & -3.94 & {$[-81.38 ;-27.26]$} & $*$ \\
\hline Age (spline 1) & 3.50 & 1.64 & 2.13 & {$[0.27 ; 6.73]$} & $*$ \\
\hline Age (spline 2) & 3.94 & 2.07 & 1.90 & {$[-0.13 ; 8.01]$} & \\
\hline Handedness & 25.51 & 19.53 & 1.31 & {$[-12.85 ; 63.87]$} & \\
\hline \multicolumn{6}{|c|}{ Computers' variables } \\
\hline $\begin{array}{l}\text { OS: OS } \\
\text { X/Windows }\end{array}$ & 4.19 & 19.95 & 0.21 & {$[-35 ; 43.37]$} & \\
\hline $\begin{array}{l}\text { OS: } \\
\text { Linux/Windows }\end{array}$ & -45.02 & 28.72 & -1.57 & {$[-101.43 ; 11.39]$} & \\
\hline $\begin{array}{l}\text { Web Browser: } \\
\text { Chrome/Firefox }\end{array}$ & -55.77 & 17.51 & -3.19 & {$[-90.16 ;-21.38]$} & $*$ \\
\hline $\begin{array}{l}\text { Web Browser: } \\
\text { IE/Firefox }\end{array}$ & 27.45 & 23.23 & 1.18 & {$[-18.19 ; 73.09]$} & \\
\hline $\begin{array}{l}\text { Web Browser: } \\
\text { Safari/Firefox }\end{array}$ & -50.50 & 29.71 & -1.70 & {$[-108.86 ; 7.86]$} & \\
\hline $\begin{array}{l}\text { Web Browser: } \\
\text { Autres/Firefox }\end{array}$ & 36.51 & 62.11 & 0.59 & {$[-85.5 ; 158.53]$} & \\
\hline
\end{tabular}

M. Longcamp

marieke.longcamp@univ-amu.fr

Aix Marseille Univ, CNRS, LPC, Marseille, France

2 Aix Marseille Univ, CNRS, BLRI, Aix-en-Provence, France

3 Aix Marseille Univ, CNRS, LNC, 3 Place Victor Hugo,

13331 Marseille Cedex 3, France 
Table 5 Mixed model regression coefficients for IKIs

\begin{tabular}{|c|c|c|c|c|c|}
\hline & $\beta$ & SE & $t$ & $95 \% \mathrm{CI}$ & (sig.) \\
\hline (Intercept) & 205.14 & 26.11 & 7.86 & & \\
\hline \multicolumn{6}{|c|}{ Experimental variables } \\
\hline Hand & -8.16 & 6.13 & -1.33 & {$[-20.21 ; 3.89]$} & \\
\hline Sequence & -21.18 & 1.65 & -12.84 & {$[-24.42 ;-17.94]$} & $*$ \\
\hline Uncertainty & -23.13 & 1.06 & -21.91 & {$[-25.2 ;-21.05]$} & $*$ \\
\hline Position & -30.81 & 1.06 & -29.18 & {$[-32.89 ;-28.74]$} & $*$ \\
\hline $\begin{array}{l}\text { Sequence } \mathrm{x} \\
\text { Uncertainty }\end{array}$ & 12.93 & 1.95 & 6.62 & {$[9.09 ; 16.77]$} & $*$ \\
\hline $\begin{array}{l}\text { Sequence } \mathrm{x} \\
\text { Position }\end{array}$ & 22.71 & 1.49 & 15.22 & {$[19.78 ; 25.64]$} & $*$ \\
\hline $\begin{array}{l}\text { Uncertainty } x \\
\text { Position }\end{array}$ & 39.30 & 1.49 & 26.37 & {$[36.38 ; 42.23]$} & $*$ \\
\hline $\begin{array}{l}\text { Sequence } \mathrm{x} \\
\text { Uncertainty } \mathrm{x} \\
\text { Position }\end{array}$ & -17.94 & 2.10 & -8.53 & {$[-22.07 ;-13.81]$} & $*$ \\
\hline Trial number & -0.28 & 0.01 & -37.89 & {$[-0.3 ;-0.27]$} & $*$ \\
\hline \multicolumn{6}{|l|}{ Subjects' variables } \\
\hline Gender & -35.56 & 6.32 & -5.63 & {$[-47.97 ;-23.16]$} & $*$ \\
\hline Age (spline 1) & 1.46 & 0.75 & 1.94 & {$[-0.02 ; 2.94]$} & \\
\hline Age (spline 2) & 2.44 & 0.95 & 2.57 & {$[0.58 ; 4.31]$} & $*$ \\
\hline Handedness & 9.41 & 8.95 & 1.05 & {$[-8.18 ; 26.99]$} & \\
\hline \multicolumn{6}{|c|}{ Computers' variables } \\
\hline $\begin{array}{l}\text { OS: OS } \\
\text { X/Windows }\end{array}$ & -1.50 & 9.14 & -0.16 & {$[-19.46 ; 16.47]$} & \\
\hline $\begin{array}{l}\text { OS: } \\
\text { Linux/Windows }\end{array}$ & -5.40 & 13.16 & -0.41 & {$[-31.26 ; 20.46]$} & \\
\hline $\begin{array}{l}\text { Web Browser: } \\
\text { Chrome/Firefox }\end{array}$ & -0.68 & 8.02 & -0.08 & {$[-16.44 ; 15.09]$} & \\
\hline $\begin{array}{l}\text { Web Browser: } \\
\text { IE/Firefox }\end{array}$ & 1.78 & 10.65 & 0.17 & {$[-19.14 ; 22.7]$} & \\
\hline $\begin{array}{l}\text { Web Browser: } \\
\text { Safari/Firefox }\end{array}$ & 0.18 & 13.62 & 0.01 & {$[-26.58 ; 26.93]$} & \\
\hline $\begin{array}{l}\text { Web Browser: } \\
\text { Autres/Firefox }\end{array}$ & 1.63 & 28.48 & 0.06 & {$[-54.32 ; 57.57]$} & \\
\hline
\end{tabular}

